\title{
A New Electrode for Electromyographic Study of Bronchial Smooth Muscle
}

\author{
Kisaburo Akasaka, * Kimio Konno, $†$ Yasuo Ono, Suetsugu \\ Mue, Chozo Abe, Mutsumi Kumagai and Tadao Ise \\ The First Department of Internal Medicine, Tohoku University \\ School of Medicine, Sendai
}

Akasaka, K., Konno, K., Ono, Y., Mue, S., Abe, C., Kumagai, M. and Ise, T. A New Electrode for Electromyographic Study of Bronchial Smooth Muscle. Tohoku J. exp. Med., 1975, 117 (1), 49-54 - The contribution of the bronchial smooth muscles to asthmatic attack has been supposed by many studies such as measurement of the intrabronchial pressure and bronchographical studies, but electromyographical evidence has not been established yet. A new bipolar platinum-ring electrode fixed on polyethylene catheter was invented in order to record action potentials from the bronchial smooth muscle through the intrabronchial lead. Action potentials obtained by this electrode were compared with those obtained by the direct peribronchial needle electrode during either artificial or spontaneous respiration in dogs and a monky. Electrophotographical analysis confirmed that action potentials obtained by our new electrode were similar in discharge pattern to those obtained by the direct peribronchial needle electrode. The active contribution of the bronchial smooth muscle to the maintenance of the bronchial caliber during spontaneous respiration was observed by our new electrode. Accordingly, this electrode is applicable to electromyographical studies of the bronchial smooth muscle. - bronchial asthma; electromyography of bronchus

As a major pathogenesis of the airway narrowing during asthmatic attack, the contraction of bronchial smooth muscles has been indirectly proposed by many studies such as the measurement of the intrabronchial pressure and bronchographical study. However, there has been no established direct evidence of contraction of the bronchial smooth muscle during asthmatic attack.

As has been proposed by Bülbring (1962), there is a close relationship between electromyographical spike potential and mechanical contraction of smooth muscle fibers.

There have been several reports (Kurata and Tsukazima 1956; Nishimine 1963; Hojyo 1964; Tozaka and Hojyo 1964; Masaoka et al. 1966) in which action potentials from the bronchial smooth muscle were measured in the experimental animals. However, their methods seem to be unapplicable to human cases.

Received for publication, March 20, 1975.

* Present address: The first Department of Internal Medicine, Fukushima Medical

College, Fukushima.

$\dagger$ Present address: Department of Central Clinical Laboratory, Akita University

School of Medicine, Akita. 
Since it is essential as a clue of pathogenesis of airway narrowing to obtain direct evidence of contraction of the bronchial smooth muscle during asthmatic attack, we have developed a new electrode which is applicable directly to the patient to record action potentials from the bronchial smooth muscle.

\section{Materials and Methods}

Figs. 1 and 2 show the structure of our new electrode used for intrabronchial lead. This electrode is a bipolar platinum-ring one, fixed on a polyethylene catheter. Nylon string is so attached to the tip of catheter that the pulling of the string makes the electrode fix closely on the internal surface of any bronchial tree, as shown in Fig. 3.

Action potentials were recorded by the direct writing oscillograph (RM-150, Nippon Kohden) and, whenever necessary, photographed by a camera (PC-1B, Nippon Kohden) fixed on the oscilloseope (VC-7, Nippon Kohden).

The electrode used for the direct peribronchial lead was the bipolar needle electrode $(40-500 \mathrm{~K} \Omega$, Sanei-sokki).

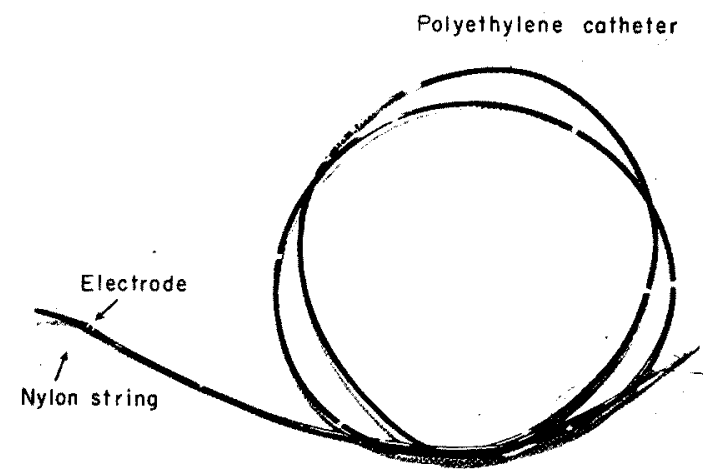

Fotw

Fig. 1. Photograph of the new electrode for intrabronchial action potential.

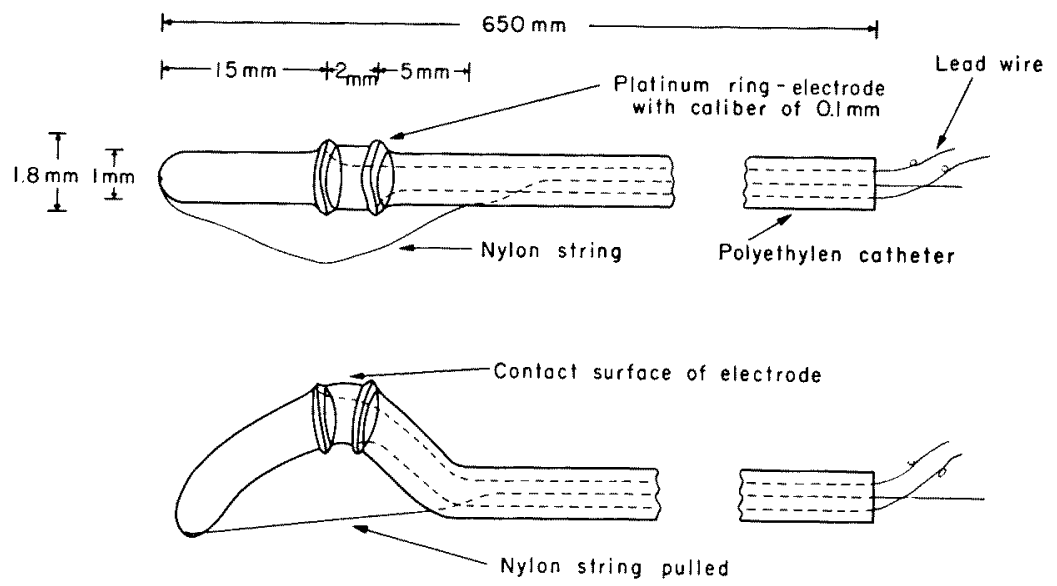

Fig. 2. Diagram of the electrode used. 


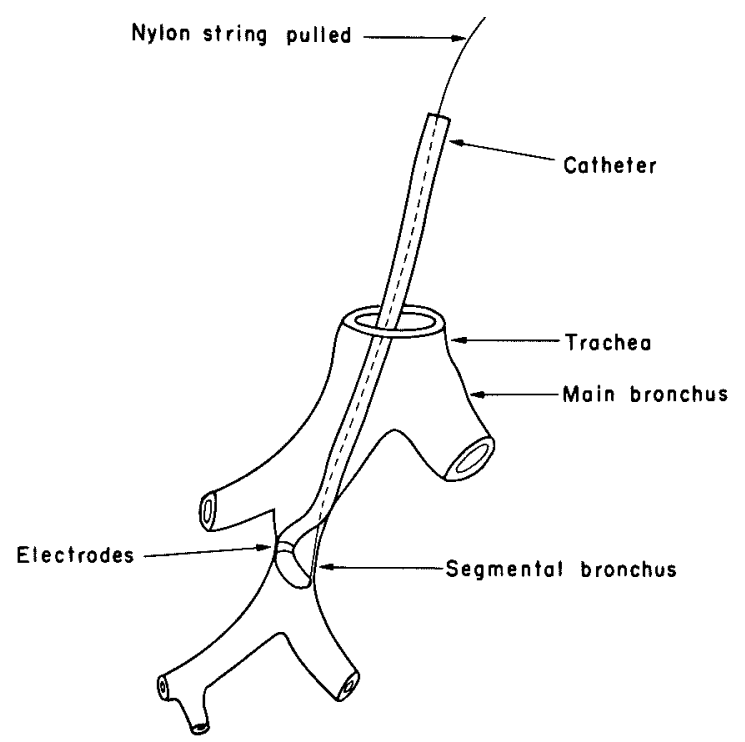

Fig. 3. Diagram of intrabronchial fixation of the electrode.

The experimental animals were one monkey $(25 \mathrm{~kg})$ and six dogs $(20-25 \mathrm{~kg})$. All animals were anesthetized by an intravenous injection of Mintal, $1.0-1.5 \mathrm{mg} / \mathrm{kg}$.

The intrabronchial as well as the peribronchial lead was made under either spontaneous or artificial respiration with the open-chest surgery. Artificial respiration was assisted by the respirator (Aica) with the appropriate ventilatory volume according to body weight.

The direct peribronchial lead was made mostly from the smooth muscle layer of the right segmental bronchus.

Ventilation was monitored by the change in the left intrapleural pressure in the case of open-chest and by the strangauge pneumograph in the spontaneous respiration.

The heart rate was monitored by electrocardiography.

The intrabronchial electrode was inserted into the bronchial tree in question via tracheal cannuls under fluoroscopical observation.

\section{Results}

\section{Peribronchial lead}

Fig. 4 shows action potentials obtained by the conventional bipolar needle electrode fixed directly on the outer surface of the bronchial muscle layer of openchest dog during artificial respiration. The amplitude of potentials ranged from 50 to $197 \mu \mathrm{V}$, and the frequency of potentials, mostly single discharge, varied considerably. Time intervals between successive discharges ranged from 0.25 to $2.4 \mathrm{sec}$, and any burst-like discharge was not observed. No relation was found between the respiratory phase and the discharge.

Fig. 5 shows action potentials of a monkey obtained in the same manner as described in Fig. 4. Action potentials of the open-chest monkey during artificial respiration were fundamentally the same as those of the dog. 


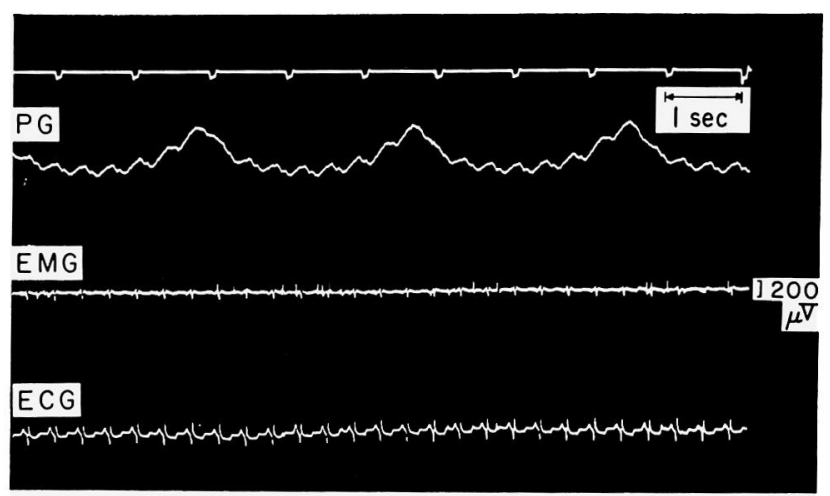

Fig. 4. Peribronchial electromyogram from an open-chest dog under artificial respiration. PG, pneumogram; EMG, electromyogram; ECG, electrocardiogram.

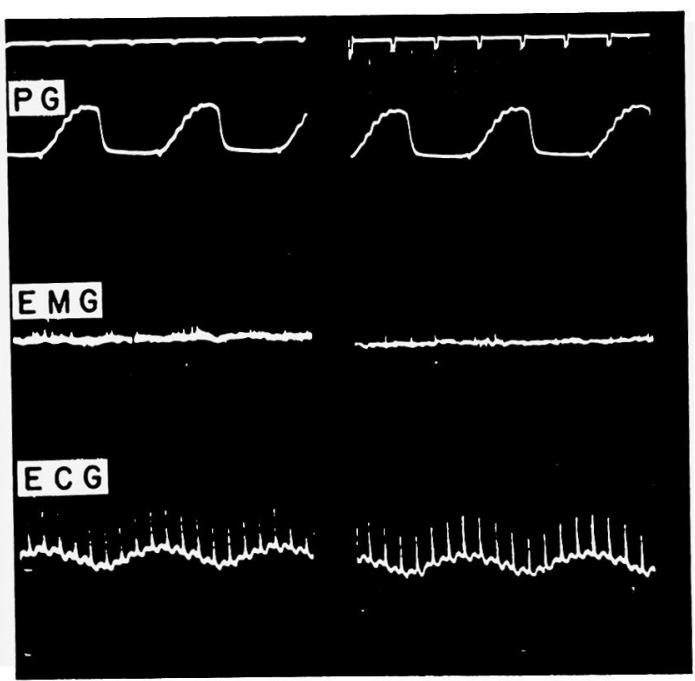

Fig. 5. Peribronchial electromyogram from an open-chest monkey under artificial respiration. PG, pneumogram; EMG, electromyogram; ECG, electrocardiogram.

\section{Intrabronchial lead}

Fig. 6 shows action potentials obtained by our new electrode closely fixed on the inner surface of the bronchus of the open-chest dog during artificial respiration.

The amplitude of potentials ranged from 62.5 to $198 \mu \mathrm{V}$ and the time interval between each successive discharges also varied from 0.2 to 3.5 sec. Action potentials were mostly single discharges with irregular frequency and occasionally 2-3 discharges were seen. No relation was observed between the respiratory phase and the discharge. No burst-like discharge was observed.

Fig. 7 shows action potentials obtained by our new electrode during spontaneous respiration in dogs. As compared with Fig. 6, the amplitude as well as 


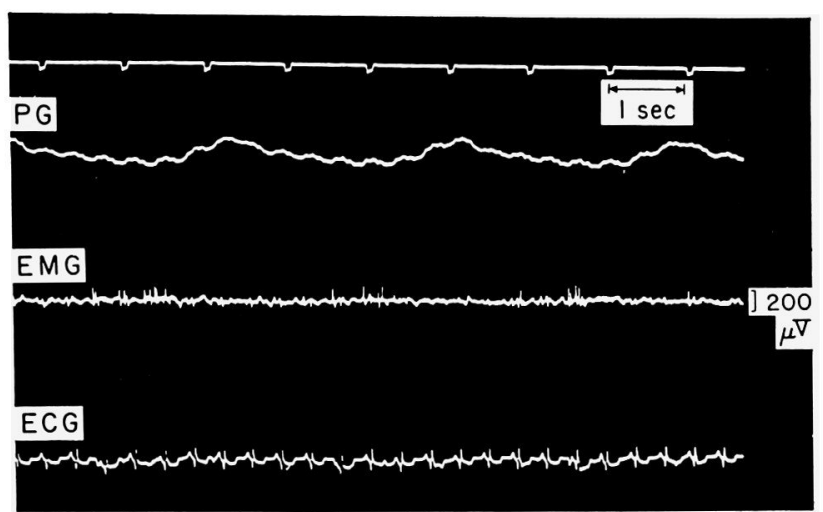

Fig. 6. Intrabronchial electromyogram from an open-chest dog under artificial respiration. PG, pneumogram; EMG, electromyogram; ECG, electrocardiogram.

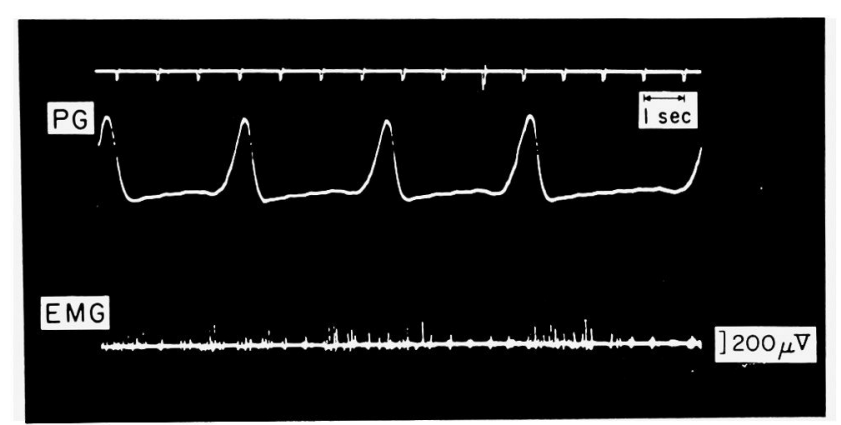

Fig. 7. Intrabronchial electromyogram in a dog under spontaneous respiration. PG, peumogram; EMG, electromyogram.

discharge frequency of action potentials slightly increased, but neither relationship between respiratory phase and discharge nor burst-like discharge was observed.

Electrophotographical analysis of these potentials showed essentially the similar pattern to that of the action potentials shown in Fig. 6.

\section{Discussion}

Several studies of the bronchial smooth muscle have been reported. All of them were based on direct peribronchial leads in the experimental animals. Kurata and Tsukazima (1956) obtained small spike potentials with amplitude of 20-50 $\mu \mathrm{V}$ and with 39-50 discharges per 10 sec in dogs. Nishimine (1963) reported action potentials with amplitude of $40-80 \mu \mathrm{V}$ and 5-20 CPS in cats. On the other hand, Tozaka and Hojyo (1964) and Hojyo (1964) obtained spike potentials with amplitude of $80-150 \mu \mathrm{V}$ and with 1-2 discharges per 5 sec irregularly, which were similar to our results. 
Generally speaking, it seems certain that discharge pattern of the bronchial smooth muscle is sporadic in the experimental animals.

Concerning the relationship between respiratory phase and discharge, we did not observe any respiratory phase dependency. Similar observations were reported by Nishimine (1963) and Hojyo (1964). On the other hand, Ellis (1936) and Drinker (1954) reported that periodical change in the bronchial caliber during ventilation was mainly produced passively by the change in the pleural pressure and there was very little electrical activity in the bronchial smooth muscle during spontaneous respiration.

However, as shown in Fig. 7, there was a slight increase in amplitude as well as in discharge frequency of action potentials obtained by intrabronchial lead during spontaneous respiration, compared with those during artificial respiration, as shown in Fig. 6. These findings are considered to show active contribution of the bronchial smooth muscle to the maintenance of the bronchial caliber during spontaneous respiration.

Electrophotographical analysis of action potentials obtained by our new intrabronchial electrode confirmed the similarity in discharge pattern to those obtained by the direct peribronchial needle electrode. Therefore, we think that our new electrode is applicable to electromyographical study of bronchial asthma.

\section{Acknowledgment}

We are sincerely thankful to Prof. Taizo Suzuki and Assist. Prof. Akinori Nishiyama, Department of Applied Physiology, Tohoku University School of Medicine, for their valuable advices.

\section{References}

1) Bülbring, E. (1962) Electrical activity in intestinal smooth muscle. Physiol. Rev., 42 Suppl. 5, 160-175.

2) Drinker, C.K. (1954) The Clinical Physiology of the Lungs, Charles C. Thomas Pub., translated by K. Tatai, Kyodo-Isho Pub. Co., Tokyo.

3) Ellis, M. (1936) The mechanism of the rhythmic changes in the caliber of the bronchi during respiration. J. Physiol. (Lond.), 87, 298.

4) Hojyo, T. (1964) Electrophysiological study of bronchial smooth muscle. $J$. physiol. Soc. (Jap.), 26, 586-599.

5) Kurata, M. \& Tsukazima, H. (1956) Effect of autonomic drugs on electromyogram of bronchial smooth muscle. Folia endocr. jap., (Jap.), 32, 208.

6) Masaoka, A., Minami, T., Maeda, I. \& Sawamura, K. (1966) Electromyogram of trachea. Jap. J. smooth Muscle Res. (Jap.), 2, 130.

7) Nishimine, Y. (1963) Studies on spasm of trachea and bronchi. Nippon Acta radiol. (Jap.), 20, 1586-1597.

8) Tozaka, T. \& Hojyo, T. (1964) Electrophysiological investigation of bronchial smooth muscle. Saishin Igaku (Jap.), 19, 3050. 